\title{
Infection of dogs with SARS-CoV-2
}

https://doi.org/10.1038/s41586-020-2334-5

Received: 12 March 2020

Accepted: 6 May 2020

Published online: 14 May 2020

Check for updates

\begin{abstract}
Thomas H. C. Sit', Christopher J. Brackman', Sin Ming Ip', Karina W. S. Tam', Pierra Y. T. Law', Esther M. W. To', Veronica Y. T. Yu', Leslie D. Sims ${ }^{2}$, Dominic N. C. Tsang ${ }^{3}$, Daniel K. W. Chu', Ranawaka A. P. M. Perera ${ }^{4}$, Leo L. M. Poon ${ }^{4} \&$ Malik Peiris ${ }^{4,5} \bowtie$
\end{abstract}

Severe acute respiratory syndrome coronavirus 2 (SARS-CoV-2) was first detected in Wuhan in December 2019 and caused coronavirus disease 2019 (COVID-19) ${ }^{1,2} \cdot \operatorname{In} 2003$, the closely related SARS-CoV had been detected in domestic cats and a dog $^{3}$. However, little is known about the susceptibility of domestic pet mammals to SARS-CoV-2. Here, using PCR with reverse transcription, serology, sequencing the viral genome and virus isolation, we show that 2 out of 15 dogs from households with confirmed human cases of COVID-19 in Hong Kong were found to be infected with SARS-CoV-2. SARS-CoV-2 RNA was detected in five nasal swabs collected over a 13-day period from a 17-year-old neutered male Pomeranian. A 2.5-year-old male German shepherd was positive for SARS-CoV-2 RNA on two occasions and virus was isolated from nasal and oral swabs. Antibody responses were detected in both dogs using plaque-reductionneutralization assays. Viral genetic sequences of viruses from the two dogs were identical to the virus detected in the respective human cases. The dogs remained asymptomatic during quarantine. The evidence suggests that these are instances of human-to-animal transmission of SARS-CoV-2. It is unclear whether infected dogs can transmit the virus to other animals or back to humans.
In Hong Kong, when a person is diagnosed with COVID-19, they are hospitalized and household contacts regarded as 'close contacts' are quarantined in designated centres. Affected pet owners are given the option of having their dogs and cats looked after and isolated by the Hong Kong Agriculture, Fisheries and Conservation Department (AFCD).Specimens are collected from these animals to assess whether they are infected with SARS-CoV-2 and to assist in determining the best methods for managing animals in quarantine, including timing of release back to the owner. Fifteen dogs and seven cats from households with known COVID-19 cases had been quarantined and tested as of 27 March 2020. During this period, two dogs returned virological test results demonstrating that they were infected.

\section{Results}

Dog 1 is a 17-year-old neutered male Pomeranian that had a number of pre-existing diseases, including a grade II heart murmur, systemic and pulmonary hypertension, chronic renal disease, hypothyroidism and a previous history of hyperadrenocorticism (F. Chan, personal communication). The owner of dog 1 was a 60 -year-old woman who developed symptoms on 12 February 2020 and was diagnosed with COVID-19 on 24 February 2020. A female domestic helper in the household developed a fever on 16 February 2020 and was subsequently confirmed to be infected with SARS-CoV-2 (secondary case A). The remaining three members of the household were sent to a quarantine centre on 26 February 2020, and one of them was confirmed to be infected on 7 March 2020 (secondary case B). Dog 1 was transferred to a holding facility managed by AFCD on 26 February 2020 and nasal, oral and rectal swabs and a faecal sample were collected. Additional specimens for virus detection were collected from the dog on six further occasions. A blood sample was collected on 3 March 2020 for serological testing (see Fig. 1). Throughout the period in quarantine, the dog remained bright and alert with no obvious change in clinical condition.

SARS-CoV-2 RNA was detected from nasal swabs collected from dog 1 by quantitative PCR with reverse transcription (RT-qPCR $)^{4,5}$ in five consecutive specimens collected on and between 26 February and 9 March 2020 (Table 1). Rectal and faecal specimens tested negative. Attempts to culture the virus from the dog were unsuccessful, probably owing to the low viral load (range $7.5 \times 10^{2}$ to $2.6 \times 10^{4}$ RNA copies per ml of specimen); in human patients with COVID-19, virus isolation had a low probability of success when viral load in the specimen was less than $10^{6}$ per $\mathrm{ml}\left(\right.$ ref. $^{6}$ ).

Dog 2 was a 2.5-year-old male German shepherd in good health from a household in which the owner developed symptoms on 10 March 2020 and was diagnosed with COVID-19 on 17 March 2020. Specimens from this dog were collected six times between 18 and 30 March 2020. Oral and nasal swabs tested positive for SARS-CoV-2 RNA on the first two occasions (Table 1). Rectal swabs collected on 18 March 2020 tested positive in four of the six assays, all with higher $C_{\mathrm{t}}$ values (lower viral load) than those obtained from oral and nasal swabs. A second dog kept in the household was sampled on four occasions between 18 and 30 March and tested negative for SARS-CoV-2 RNA in all tests.

Serum samples collected from dog 1 on 3 March 2020, and from dog 2 on 19, 23 and 30 March 2020 were tested for SARS-CoV-2 antibody using $90 \%$ plaque-reduction neutralization tests $\left(\mathrm{PRNT}_{90}\right)^{7}$. Serum from $\operatorname{dog} 1$ had a PRNT 90 titre of 1:80; serum from dog 2 had PRNT $_{90}$ titres of

${ }^{1}$ Agriculture, Fisheries and Conservation Department, Government of the Hong Kong SAR, Hong Kong Special Administrative Region, Hong Kong, China. ${ }^{2}$ Asia Pacific Veterinary Information Services, Melbourne, Victoria, Australia. ${ }^{3}$ Public Health Laboratory Centre, Centre for Health Protection, Department of Health, Government of the Hong Kong SAR, Hong Kong Special Administrative Region, Hong Kong, China. ${ }^{4}$ School of Public Health, The University of Hong Kong, Hong Kong Special Administrative Region, Hong Kong, China. ${ }^{5} \mathrm{HKU}$-Pasteur Research Pole, The University of Hong Kong, Hong Kong Special Administrative Region, Hong Kong, China. ${ }_{\text {e-mail: malik@hku.hk }}$ 


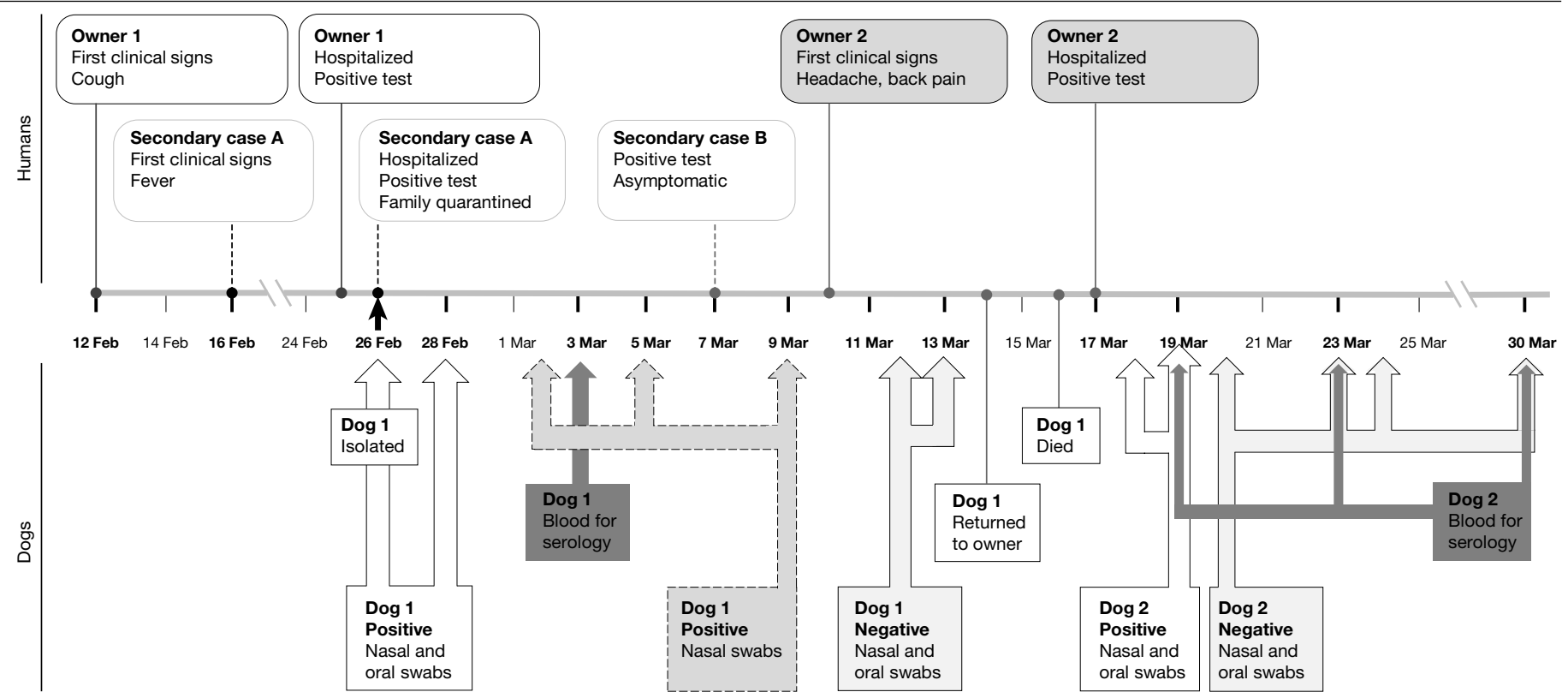

Fig. 1 Timeline. A timeline of clinical events in the human and dog SARS-CoV-2 infection cases that were analysed in this study.

1:10 (19 March), 1:40 (23 March) and 1:160 (30 March). The second dog in the household of dog 2 remained antibody-negative on 30 March 2020. Twenty control dog sera tested negative for $\mathrm{PRNT}_{90}$-neutralizing antibody.

Viral RNA from the nasal swab specimen collected from dog 1 on 26 and 28 February 2020 was sequenced directly from the clinical specimen and compared with the virus found in clinical specimens from the owner and secondary cases $A$ and $B$. The full virus genome sequence $(29,764$ nucleotides) was obtained from the index case and from secondary cases A and B. Viral sequences of length 27,871 nucleotides (nt) ( $94 \%$ of the genome) and $26,025 \mathrm{nt}$ ( $93 \%$ of the genome) were obtained from the nasal swabs of dog 1 collected on 26 and 28 February 2020 , respectively. The viral sequences from the index case and two secondary cases were identical across the full genome. Viral RNA from the nasal swabs of dog 2 collected on 18 and 19 March 2020 and the human index case from the same household were sequenced and found to be identical across the full genome (29,764 nucleotides). The viruses from the two households, however, were clearly distinguishable (Fig. 2).

\section{Discussion}

Our results demonstrate infection of two dogs by SARS-CoV-2. Angiotensin-converting enzyme 2 (ACE2) is known to be the human receptor for SARS-CoV-2, and canine ACE2 is similar to that of humans

Table 1 | RT-qPCR testing results on nasal and oral swabs of the dogs and serology

\begin{tabular}{|c|c|c|c|c|c|c|c|c|c|c|c|c|c|c|c|}
\hline & \multirow[b]{3}{*}{$\begin{array}{l}\text { Date of } \\
\text { collection }\end{array}$} & \multicolumn{4}{|c|}{ TLVL laboratory } & \multicolumn{10}{|c|}{ HKU laboratory } \\
\hline & & \multicolumn{2}{|l|}{$C_{t}(E)$} & \multicolumn{2}{|c|}{$\mathrm{C}_{\mathrm{t}}(\mathrm{RdRp})$} & \multicolumn{2}{|c|}{$C_{t}(n s p 14)$} & \multicolumn{3}{|c|}{$C_{t}(N)$} & \multicolumn{2}{|c|}{$C_{t}(n s p 16)$} & \multicolumn{2}{|l|}{$C_{t}(M)$} & \multirow{2}{*}{$\begin{array}{l}\text { Serum } \\
\text { PRNT }_{90} \text { titre }\end{array}$} \\
\hline & & Nasal & Oral & Nasal & Oral & Nasal & Oral & Nasal & $\begin{array}{l}N \text { gene copies } \\
\text { per ml (nasal) }\end{array}$ & Oral & Nasal & Oral & Nasal & Oral & \\
\hline \multirow{8}{*}{$\begin{array}{l}\text { Dog } 1 \\
\text { (potential } \\
\text { exposure } \\
12-26 \\
\text { Feb) }\end{array}$} & 26 Feb & 33.90 & 34.52 & 38.97 & Neg. & 36.76 & 37.96 & 34.71 & 11,741 & 36.48 & 37.94 & 39.25 & 36.91 & 37.95 & \\
\hline & $28 \mathrm{Feb}$ & 31.98 & Neg. & 37.44 & Neg. & 38.96 & 39.01 & 34.58 & 10,145 & Neg. & 38.64 & Neg. & 38.97 & Neg. & \\
\hline & 2 Mar & 31.69 & Neg. & Neg. & Neg. & 32.49 & Neg. & 33.2 & 25,788 & Neg. & 32.71 & Neg. & 32.41 & Neg. & \\
\hline & 3 Mar & & & & & & & & & & & & & & $1: 80$ \\
\hline & 5 Mar & 33.58 & Neg. & 38.53 & Neg. & 39.14 & Neg. & 38.43 & 751 & Neg. & 37.72 & Neg. & Neg. & Neg. & \\
\hline & 9 Mar & 30.07 & Neg. & Neg. & Neg. & 35.86 & Neg. & 34.97 & 7,777 & Neg. & 36.96 & Neg. & 36.24 & Neg. & \\
\hline & $12 \mathrm{Mar}$ & Neg. & Neg. & Neg. & Neg. & Neg. & Neg. & Neg. & Neg. & Neg. & Neg. & Neg. & Neg. & Neg. & \\
\hline & 13 Mar & Neg. & Neg. & Neg. & Neg. & Neg. & Neg. & Neg. & Neg. & Neg. & Neg. & Neg. & Neg. & Neg. & \\
\hline \multirow{6}{*}{$\begin{array}{l}\text { Dog } 2 \\
\text { (potential } \\
\text { exposure } \\
10-17 \\
\text { Mar) }\end{array}$} & $18 \mathrm{Mar}$ & 24.85 & 26.60 & 31.19 & 32.63 & 26.74 & 28.72 & 27.31 & 724,500 & 29.33 & 28.26 & 30.29 & 27.73 & 29.49 & \\
\hline & $19 \mathrm{Mar}$ & 28.11 & 31.23 & 36.12 & 38.45 & 32.98 & 36.09 & 32.66 & 62,933 & 36.98 & 33.65 & 36.95 & 32.17 & 35.97 & $<1: 10$ \\
\hline & $20 \mathrm{Mar}$ & Neg. & Neg. & Neg. & Neg. & Neg. & Neg. & Neg. & Neg. & Neg. & Neg. & Neg. & Neg. & Neg. & \\
\hline & $23 \mathrm{Mar}$ & Neg. & Neg. & Neg. & Neg. & Neg. & Neg. & Neg. & Neg. & Neg. & Neg. & Neg. & Neg. & Neg. & $1: 40$ \\
\hline & $24 \mathrm{Mar}$ & Neg. & Neg. & Neg. & Neg. & Neg. & Neg. & Neg. & Neg. & Neg. & Neg. & Neg. & Neg. & Neg. & \\
\hline & 30 Mar & Neg. & Neg. & Neg. & Neg. & Neg. & Neg. & Neg. & Neg. & Neg. & Neg. & Neg. & Neg. & Neg. & $1: 160$ \\
\hline Cut-off $C_{t} f$ & for positive & $<36$ & $<36$ & $<39$ & $<39$ & $<40$ & $<40$ & $<40$ & $<40$ & $<40$ & $<40$ & $<40$ & $<40$ & $<40$ & \\
\hline
\end{tabular}

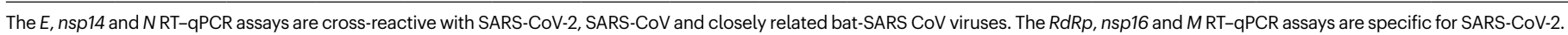
Gene copies per millilitre of original swab specimen with adjustment for virus extraction dilutions. Assumes no pre-symptomatic shedding of virus from human cases. Neg., negative. 


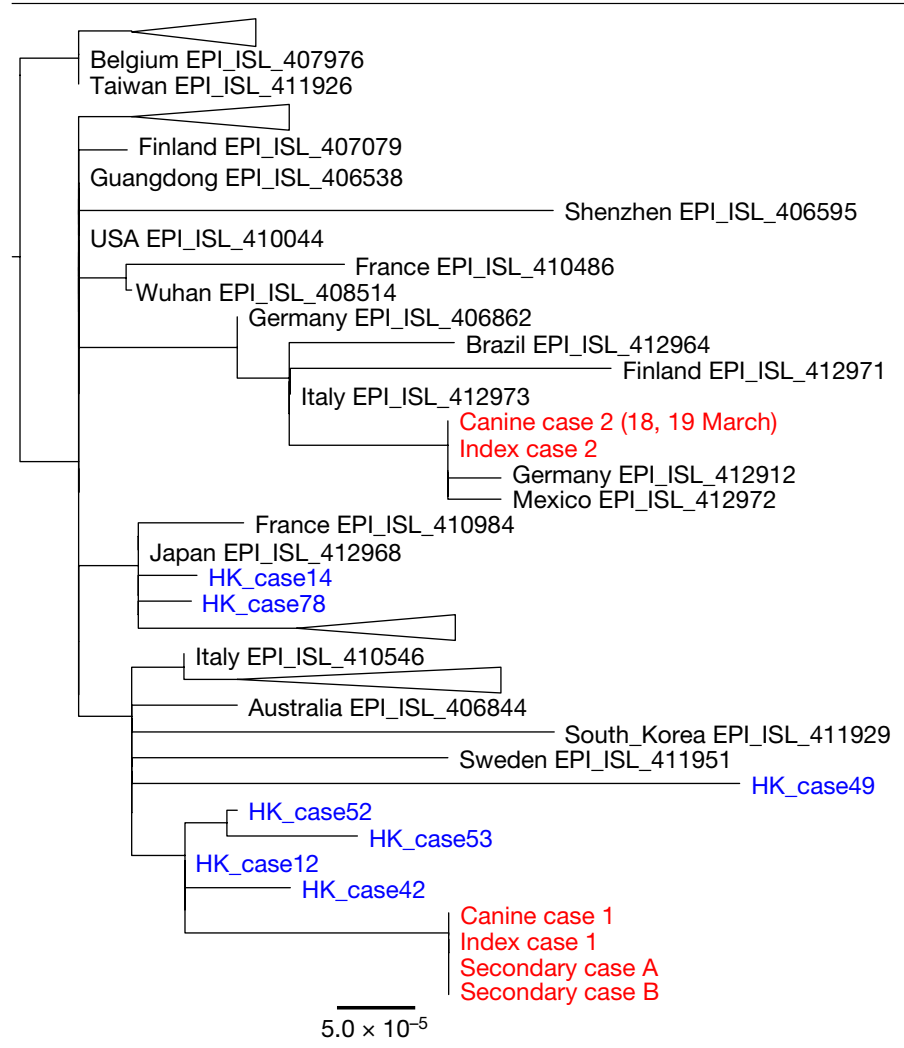

Fig. 2 | A phylogenetic tree of SARS-CoV-2 showing viruses from infected dogs and humans in Hong Kong. Virus sequences from humans and dogs from the two affected households are shown in red. Other virus sequences from human patients in Hong Kong are shown in blue. Selected full and partial (longer that 23,000 nt) virus genomes from the GISAID database are included in this analysis. The tree is unrooted and was constructed using the maximum-likelihood method with PhyML.

(Extended Data Fig. 1). Of the 18 amino acids known to be involved in the interaction between $\mathrm{ACE} 2$ and the spike receptor binding domain (RBD) of SARS-CoV-2, five differ between humans and dogs, but none of these are in regions known to disrupt the interaction between the RBD of SARS-CoV and ACE2 ${ }^{8}$.

Our evidence suggests that human-to-animal transmission of SARS-CoV-2 is possible. We do not have information on whether the virus can cause illness in dogs, but there were no specific symptoms in either of the infected dogs while they were shedding virus. The Pomeranian died two days after release from isolation, probably owing to the pre-existing underlying diseases; we were unable to perform a post mortem examination. Whether infected dogs could transmit the virus to other animals or back to humans remains unknown. The owner of dog 2 had a second, crossbreed dog in which neither viral RNA nor antibody responses were detected, suggesting that transmission had not occurred between the two dogs sharing the household.

These two cases in Hong Kong demonstrate that dogs can acquire infection in households with SARS-CoV-2-infected humans. A survey of 4,000 specimens from dogs, cats and horses from places where community transmission of SARS-CoV-2 was occurring in humans did not detect any positive results, suggesting that the virus is not widely circulating in pet animals ${ }^{9}$. Unlike our study, this previous study did not specifically investigate dogs from households of patients with COVID-19. A challenge study in five six-week-old beagles demonstrated seroconversion in two dogs and detection of viral RNA (up to $10^{6.5}$ copies) in rectal swabs two days after challenge, and one dog had viral RNA in a rectal swab six days after challenge. No virus was detected in oropharyngeal swabs, but nasal swabs were not collected ${ }^{10}$. Our results suggest higher viral load and increased duration of viral shedding in nasal swabs compared with oral swabs. The experimental challenge study reported that cats had large quantities of virus in nasal mucosa and other tissues, and that they shed sufficient virus to allow cat-to-cat transmission ${ }^{10}$. A cat that was in contact with a human patient with COVID-19 tested positive for SARS-CoV-2 in Belgium ${ }^{11}$.SARS-CoV-2 RNA was detected in a cat in Hong Kong after the cut-off date for the present study; the cat was from a household with a confirmed case of COVID-19.

These findings and the results from animal testing during the SARS outbreak in $2003^{3}$ have potential implications for the management of mammalian pets owned by people who develop SARS-CoV-2 infection. There is no evidence that domestic animals had any role in onward transmission of the SARS outbreak ${ }^{3}$. However, from a precautionary point of view, pets belonging to patients with COVID-19 could be isolated and tested for SARS-CoV-2, as is being done in Hong Kong.

The findings also have implications for future zoonotic transmission events by the precursor virus of SARS-CoV-2. Rhinolophid bats are considered a probable reservoir of the precursor of SARS-CoV- $2^{12}$. However, on the basis of experiences with SARS virus, intermediate hosts probably serve to bridge transmission from bats to humans. Dogs, other canids and felids can be sold in or present in the vicinity of wild-game animal markets, the presumed source for the initial zoonotic spillover of SARS-CoV-2. Studies into the origin of SARS-CoV-2 should investigate these species to determine whether they have any role in spillover events.

\section{Online content}

Any methods, additional references, Nature Research reporting summaries, source data, extended data, supplementary information, acknowledgements, peer review information; details of author contributions and competing interests; and statements of data and code availability are available at https://doi.org/10.1038/s41586-020-2334-5.

1. Zhu, N. et al. A novel coronavirus from patients with pneumonia in China, 2019. N. Engl. J. Med. 382, 727-733 (2020).

2. World Health Organization. Coronavirus Disease 2019 (COVID-19) Situation Report 50 (2020); https://www.who.int/docs/default-source/coronaviruse/situation-reports/202003 10-sitrep-50-covid-19.pdf?sfvrsn=55e904fb_ 2

3. World Health Organization. Consensus Document on the Epidemiology of Severe Acute Respiratory Syndrome (SARS) (2003); https://apps.who.int/iris/bitstream/ handle/10665/70863/WHO CDS CSR_GAR 2003.11 eng.pdf

4. Chu, D. K. W. et al. Molecular diagnosis of a novel coronavirus (2019-nCoV) causing an outbreak of pneumonia. Clin. Chem. 66, 549-555 (2020).

5. Corman, V. M. et al. Detection of 2019 novel coronavirus (2019-nCoV) by real-time RT-PCR. Euro Surveill. 25 (2020).

6. Wölfel $R$, et al. Virological assessment of hospitalized patients with COVID-2019. Nature 581, 465-469 (2020).

7. Choe, P. G. et al. MERS-CoV antibody responses 1 year after symptom onset, South Korea, 2015. Emerg. Infect. Dis. 23, 1079-1084 (2017).

8. Lan J et al. Structure of the SARS-CoV-2 spike receptor-binding domain bound to the ACE2 receptor. Nature 581, 215-220 (2020).

9. IDEXX. IDEXX SARS-CoV-2 (COVID-19) RealPCR Test (2020); https://www.idexx.com/en/ veterinary/reference-laboratories/idexx-sars-cov-2-covid-19-realpcr-test/

10. Shi, J. et al. Susceptibility of ferrets, cats, dogs, and other domesticated animals to SARS-coronavirus 2. Science 368, 1016-1020 (2020).

11. SciCom. Risque Zoonotique du SARS-CoV2 (Covid-19) Associé aux Animaux de Compagnie: Infection de l'Animal vers l'Homme et de l'Homme vers l'Animal (2020); http://www.afsca.be/comitescientifique/avis/2020/documents/ Conseilurgentprovisoire04-2020_SciCom2020-07_Covid-19petitsanimauxdomestiqu es_27-03-20_001.pdf

12. Zhou, P. et al. A pneumonia outbreak associated with a new coronavirus of probable bat origin. Nature 579, 270-273 (2020).

Publisher's note Springer Nature remains neutral with regard to jurisdictional claims in published maps and institutional affiliations.

(c) The Author(s), under exclusive licence to Springer Nature Limited 2020 


\section{Methods}

\section{Data reporting}

No statistical methods were used to predetermine sample size. The experiments were not randomized. The investigators were not blinded to allocation during experiments and outcome assessment.

\section{Specimen collection}

Specimens from dogs and cats were collected by veterinarians from animals sent to the AFCD isolation centre and included deep oropharyngeal and nasal swabs and a sample of fresh faeces and/or a rectal swab, placed in virus transport medium and kept on cool-packs until arrival in the laboratory. Virus transport medium comprised Medium 199 (Sigma M0393) as basal medium, $0.5 \%$ bovine serum albumin, antibiotics (penicillin $g$, streptomycin sulfate, polymyxin B sulfate, sulfamethoxazole, nystatin, gentamicin sulfate, ofloxacin). Specimens were collected on at least 3 occasions (on arrival in the isolation centre and in the two days before release). Any animal that had a positive test was retested until no positive results were obtained. Owners provided written consent at the time their pets were moved to isolation to allow specimens to be collected and tested.

Control specimens including nasal, oral, rectal swabs and faeces were collected from 21 stray dogs soon after euthanasia. Stored residual sera from 20 dogs collected for diagnostic purposes from veterinary clinics during 2017-2018 were used as controls for serology.

Specimens from humans were collected and tested by RT-qPCR as part of routine clinical care and the viruses genetically sequenced as part of the routine public health response (Institutional Review Board approval UW20-168).

\section{Quantitative RT-PCR}

At the AFCD laboratory, RNA from $200 \mu \mathrm{l}$ specimen in virus transport medium was extracted using NucliSENS easyMag extraction kit (BioMerieux) following instructions provided by the manufacturer and eluted into $60 \mu \mathrm{l}$. The RNA was tested for SARS-CoV-2 RNA in a commercial assay RT-qPCR assay for the $E$ and $R d R p$ gene sequences (TIB Molbiol Lightmix Modular Assays) based on published RT-qPCR assay for SARS-CoV-2 ${ }^{5}$. Positive, negative and inhibitor controls were included in each RT-qPCR run and work-flow precautions were in place to minimise PCR contamination. Positive samples were sent to the HKU as an independent reference laboratory for confirmation.

Viral RNA from the original swabs referred by the AFCD laboratory were independently extracted at the HKU using the QIAamp viral RNA minikit (Qiagen, Hilden, Germany) following the manufacturer's instructions. Swab supernatant $(160 \mu \mathrm{l})$ was used for RNA extraction with the final elution volume being $60 \mu \mathrm{l}$. One-step RT-qPCR assays were run for previously published $n s p 14$ and $N$ genes, which detect SARS-CoV, SARS-CoV-2 and bat SARS-CoV ${ }^{4}$. In addition, RT-qPCR assays for $n s p 16$ and $M$ that are specific for SARS-CoV-2 with no cross-reaction with SARS-CoV were also used. The forward primer (5'-GGWCAAATCAATGATATGATTTT), reverse prime (5'-GTTGTTAACAAGAACATCACTAGA) and probe (5'-FAM-AAGTCTRCCTTTACTAAGAAGAGA-TAMRA-3') were used for the ORF1b-nsp16 assay and forward primer (5'-GGYTCTAARTCACCCA TTCA-3'), reverse prime ( $5^{\prime}$-TGATACTCTARAAAGTCTTCATA-3 $\left.{ }^{\prime}\right)$ and probe (5'-FAM-AATTTAGGTTCCTGGCAATTAATT-TAMRA-3') were used for the $M$ gene assay. The thermal cycling conditions were identical to those published for the $n s p 14$ and $N$ gene assays ${ }^{4}$. Positive, negative and inhibitor controls were included in each RT-qPCR run and work-flow precautions were in place to minimise PCR contamination ${ }^{13}$.

Nasal, oral, rectal swabs and faecal samples from 21 control dogs were run by all six RT-qPCR assays with negative results. No evidence of PCR inhibition was seen in any of these RNA extracts.

\section{Sequencing the viral genomes}

To amplify the virus genome, reverse transcription reactions were set up using superscript IV reverse transcriptase (Thermo Fisher Scientific) with multiple gene specific primers targeting different regions of the viral genome (Supplementary Table). The synthesized cDNA was then subjected to multiple overlapping PCRs using Platimum Taq DNA polymerase (Thermo Fisher Scientific) using the protocol provided by the manufacturer. The PCRs performed were in sizes of around 2,000 bp designed to cover the whole virus genome. PCR amplicons were visualized by agarose gel electrophoresis. Nested PCRs were performed when necessary for genome amplification. Aliquots of $5 \mu \mathrm{l}$ PCR products and DNA ladder were loaded into wells in $2 \%$ agarose gel. Electrophoresis was run at $120 \mathrm{~V}$ for $20 \mathrm{~min}$ in TAE buffer and the DNA band was visualized with SYBR safe DNA gel stain.

PCR amplicons obtained from the same specimens were pooled and sequenced using MiSeq sequencing platform (Illumina). Sequencing library was prepared by Nextera XT DNA library prep Kit (Illumina) following standard protocols. Generated sequencing reads were mapped to a reference virus genome by $\mathrm{BWA}^{14}$ and genome consensus was generated by Geneious version 11.1.4 (https://www.geneious.com) with a minimal coverage depth of 20. Percentage of nucleotides at each position of the genome was calculated by bam-readcount (https://github. com/genome/bam-readcount) with minimal base quality score of 20 and minimum mapping quality score of 20.

\section{Plaque reduction neutralization tests}

BetaCoV/Hong Kong/VM20001061/2020 isolated from the nasopharynx aspirate and throat swab of a COVID-19 patient in Hong Kong was grown in Vero E6 cells (ATCC CRL-1586). Cells were regularly tested to exclude mycoplasma contamination. Stock virus was prepared and aliquoted and stored at $-80^{\circ} \mathrm{C}$ until use. The virus stock was titrated in quadruplicate in Vero-E6 cells in 24-well tissue culture plates (TPP Techno Plastic Products) in a biosafety level 3 facility. After one hour incubation in $5 \% \mathrm{CO}_{2}$ incubator, the plates were overlaid with $1 \%$ agarose in cell culture medium and incubated for 3 days when the plates were fixed and stained and plaque forming units per $\mathrm{ml}$ of the virus stock was determined. Serial dilutions of serum samples were then incubated with 30-40 plaque-forming units of virus for $1 \mathrm{~h}$ at $37^{\circ} \mathrm{C}$. The virus-serum mixtures were added on to Vero cell monolayers, incubated, overlaid and stained as above. Antibody titres were defined as the highest serum dilution that resulted in $>90 \%\left(\mathrm{PRNT}_{90}\right)$ reduction in the number of plaques ${ }^{7}$.

\section{Virus isolation}

Fresh nasal and oral swab fluid collected from SARS-CoV-2 PCR confirmed dogs in viral transport media were used as the inoculum for virus isolation. In brief, Vero E6 (ATCC CRL-1586) cells were cultured for $24 \mathrm{~h}$ in a 24-well plate format (TPP Techno Plastic Products) before inoculation. Culture medium was minimal essential medium

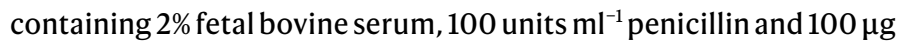
$\mathrm{ml}^{-1}$ streptomycin. The swab fluids were centrifuged at 5,000 rpm for $10 \mathrm{~min}$ at $4{ }^{\circ} \mathrm{C}$ in a benchtop centrifuge and the supernatant was separated and inoculated on to Vero E6 cells in alternative wells of the 24-well plate. After two hours incubation for adsorption in a $37^{\circ} \mathrm{C}$ incubator containing $5 \% \mathrm{CO}_{2}$, fresh virus growth medium was added to a final volume of $1 \mathrm{ml}$ and then incubated in a $37^{\circ} \mathrm{C}$ incubator containing $5 \% \mathrm{CO}_{2}$ for six days. The presence of cytopathic effect (CPE) was looked for daily. Additionally, the aliquots of culture supernatant samples was collected into AVL buffer at $\mathrm{Oh}$, $24 \mathrm{~h}, 48 \mathrm{~h}$ and $72 \mathrm{~h}$ post inoculation for PCR. The culture medium was replaced as required with fresh culture medium. Cell cultures that were negative for virus growth were blind-passaged again after six days. The cultures that were positive for virus growth as judged by cytopathic effect and increasing viral load by RT-qPCR were collected and passed on to new cull culture wells in 24-well plates and then progressively onto cells in T25 culture flasks (Greiner Bio-one). Mock inoculated Vero E6 cells were used as negative control for each isolation experiment. 


\section{Article}

\section{Reporting summary}

Further information on research design is available in the Nature Research Reporting Summary linked to this paper.

\section{Data availability}

Data that support the findings of this study have been deposited at GenBank with accession numbers MT215193, MT215194, MT215195, MT270814, MT270815 and MT276600. The sequencing primers used for full genome sequencing of SARS-CoV-2 are available in the Supplementary Table.

13. Bustin S.A. et al. MIQE précis: practical implementation of minimum standard guidelines for fluorescence-based quantitative real-time PCR experiments. BMC Mol. Biol. 11, 74 (2010).

14. Li, H. \& Durbin, R. Fast and accurate short read alignment with Burrows-Wheeler transform. Bioinformatics 25, 1754-1760 (2009).

Acknowledgements We acknowledge the work by staff of the Hong Kong SAR Centre for Health Protection who undertook investigations of the human cases in the affected household. We thank the dog owners for allowing the material on these cases to be published. We thank E. Tai for information on infection in pet animals during the SARS outbreak; E. Yiu for design and preparation of the timeline; H.-L. Yen for providing control canine sera; P. Krishnan and D. Yuet Mei Ng for technical assistance; the core facility at the Centre for PanorOmic Sciences at the University of Hong Kong for the Illumina MiSeq sequencing of viral genomes; and the originating and submitting laboratories for sharing genetic sequences and other associated data through the GISAID Initiative for SARS-CoV-2 genome sequences. We acknowledge research funding for M.P. from the US National Institute of Allergy and Infectious Diseases (NIAID) under Centers of Excellence for Influenza Research and Surveillance (CEIRS) contract no. HHSN272201400006C.

Author contributions T.H.C.S., E.M.W.T., M.P., L.D.S. and C.J.B. were responsible for design of the study. Monitoring and collection of samples from dogs was undertaken by E.M.W.T. and V.Y.T.Y., and data and samples from humans was curated by D.N.C.T. Molecular diagnostics was undertaken and overseen by P.Y.T.L., S.M.I., K.W.S.T. and D.K.W.C. Virus genetic sequencing was undertaken by D.K.W.C., L.L.M.P. and M.P. L.D.S. and T.H.C.S. drafted the manuscript. Data analysis and critical review of the manuscript was undertaken by all authors.

Competing interests The authors declare no competing interests.

\section{Additional information}

Supplementary information is available for this paper at https://doi.org/10.1038/s41586-0202334-5.

Correspondence and requests for materials should be addressed to M.P.

Peer review information Nature thanks Linda Saif and the other, anonymous, reviewer(s) for their contribution to the peer review of this work.

Reprints and permissions information is available at http://www.nature.com/reprints. 


Human ACE2
Dog ACE2
Macaca ACE2
Masked palm civet ACE2
Cat ACE2
Mouse ACE2

Human ACE2
Dog ACE2
Macaca ACE2
Masked palm civet ACE2
Cat ACE2
Mouse ACE2

Human ACE2
Dog ACE2
Macaca ACE2
Masked palm civet ACE2
Cat ACE2

ACE2

Mouse ACE2

10 20

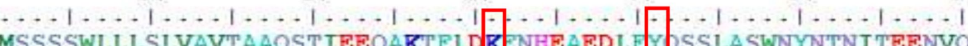
MSSSSWLLLSLVAVTAAQST IEEQARTELDRENHEAEDLE Y SSSLASWNYNTNITEENVQ

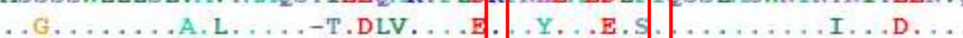

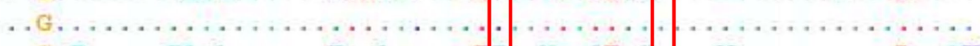

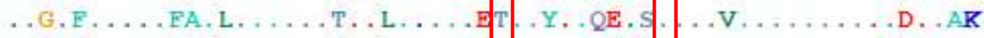

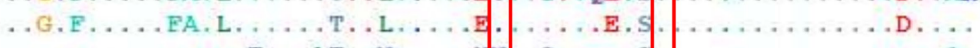

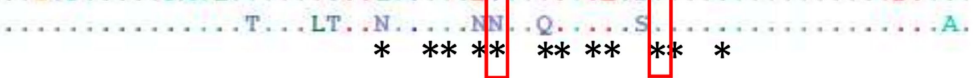

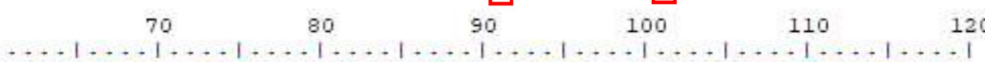
NMNNAGDKWSAFLKEQSTLAQMYPLQEIQNLTVRLQLQALQQNGSSVLSEDKSKRLNTIL

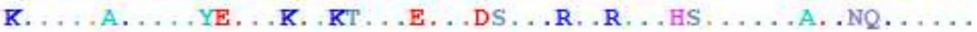
$\ldots \ldots$ E. . . . . . . . . . . . . . . . . . . . . . . .

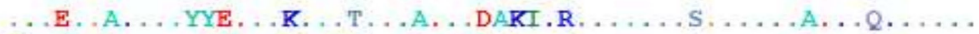

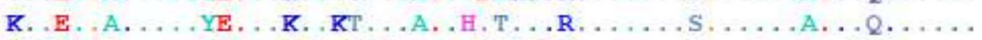

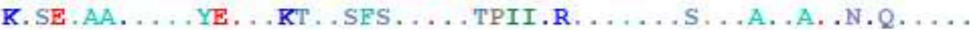
$* *$

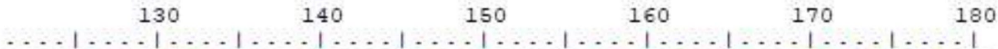
NTMSTIYSTG KVCNPDNPQECLLLEPGLNEIMANSLDYNERLWAWESWRSEVGKQLRPLY .S. . . . . . A. . . . . . . . . . . DD . E. . K. . . . . . . . . . . . . $\ldots \ldots \ldots \ldots$. . . . . . D . . . ER . . . . . . . . . . . . . .

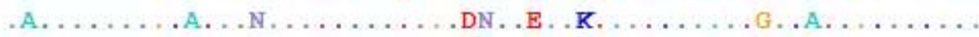
A. . . . . . A. . N . . . . . . DD. . . . K. . . . . . . A. . . . .

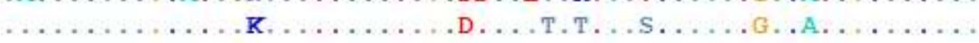

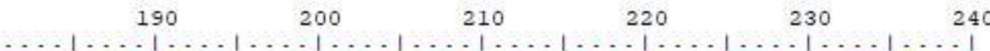
EEYVVLKNEMARAN--HYEDYGDYWRGDYEVNGVDGYDYSRGQLIEDVEHTEEEIRPLYE ....A. . . . . . - N . . . . . . . EEWEN . . . .N . . D . . . . TQ.M. . Q

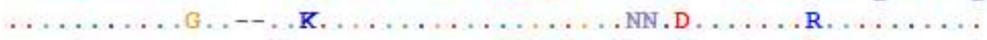

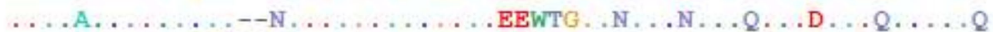

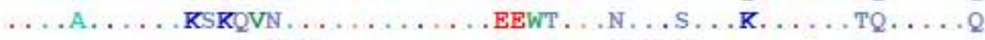

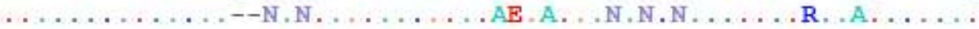

Mouse $A C P^{2}$

Human ACE2
Dog ACE2
Macaca ACE2
Masked palm civet ACE2
Cat ACE2
Mouse ACE2

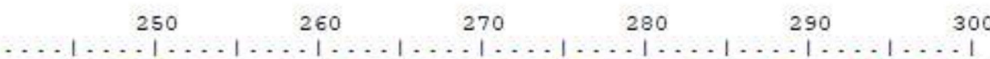
HLHAYVRAKLMNAYPSYISPIGCLPAHLLGDMWGRFWTNLYSLTVPFGORPNIDVTDAMV

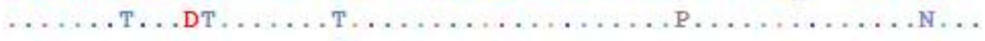

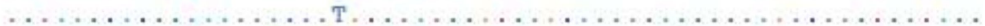

$\ldots \ldots \ldots$. DT . R. RT . . . . . . . . . . . . . . . . . . . . . . . . $\ldots \ldots \ldots \ldots$ DT . . R . . T. . . . . . . . . . . . . . . . . .

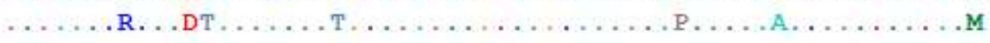

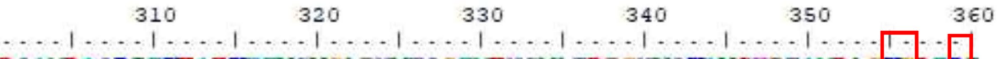
DQAWDAQRIE KEAEKEEVSVGLPNMTQGEWENSMLTDP GNVQRAVCHPTAWDL R PER N.S. .RR. . . . . . . . . . E. . . . . E.SDSR.V . . . . . . . .

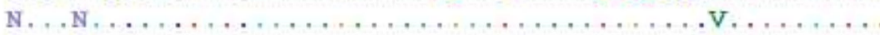

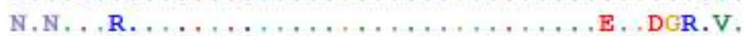
N.S. R. . . . . . . . . . . . . . . . . .

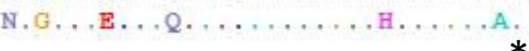

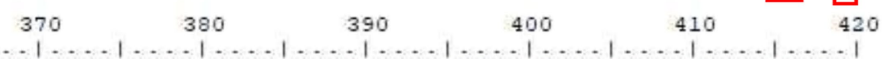
Human ACE2 Dog ACE2 Macaca ACE2 Masked palm civet ACE2 Cat ACE2

Mouse ACE2

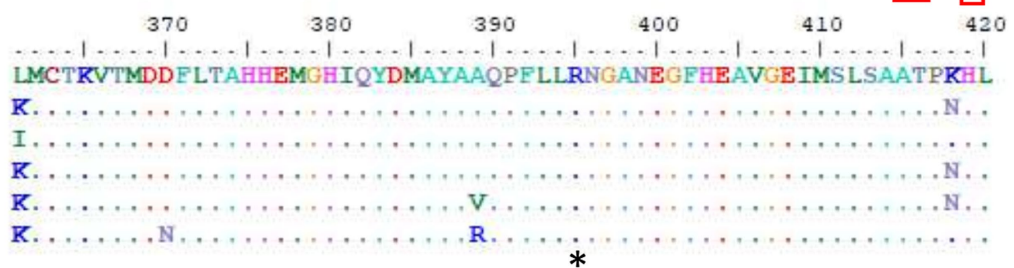

Extended Data Fig. 1 | Sequence alignment of ACE2 proteins from human, dog, macaque, masked palm civet, cat and mouse. Amino acid residues of human ACE2 that are experimentally shown to interact with the RBD of SARS-CoV- ${ }^{8}$ are denoted by asterisks. Mutations known to disrupt the interaction between human ACE2 and RBD of SARS-CoV are highlighted in red boxes and these amino acid residues are all conserved between human and dog ACE2 proteins. 


\section{Reporting Summary}

Nature Research wishes to improve the reproducibility of the work that we publish. This form provides structure for consistency and transparency in reporting. For further information on Nature Research policies, seeAuthors \& Referees and theEditorial Policy Checklist .

\section{Statistics}

For all statistical analyses, confirm that the following items are present in the figure legend, table legend, main text, or Methods section. $\mathrm{n} / \mathrm{a} \mid$ Confirmed

$\boldsymbol{x} \square$ The exact sample size $(n)$ for each experimental group/condition, given as a discrete number and unit of measurement

$\mathbf{x} \square$ A statement on whether measurements were taken from distinct samples or whether the same sample was measured repeatedly

$\mathbf{x} \square$ The statistical test(s) used AND whether they are one- or two-sided

$\boldsymbol{x} \square$ Only common tests should be described solely by name; describe more complex techniques in the Methods section.

$\boldsymbol{x} \square$ A description of all covariates tested

$\boldsymbol{x} \square$ A description of any assumptions or corrections, such as tests of normality and adjustment for multiple comparisons

$\mathbf{x} \square$ A full description of the statistical parameters including central tendency (e.g. means) or other basic estimates (e.g. regression coefficient)

$x-$ AND variation (e.g. standard deviation) or associated estimates of uncertainty (e.g. confidence intervals)

$\mathbf{x} \square$ For null hypothesis testing, the test statistic (e.g. $F, t, r$ ) with confidence intervals, effect sizes, degrees of freedom and $P$ value noted

$\boldsymbol{x} \square$ Give $P$ values as exact values whenever suitable.

$\mathbf{x} \square$ For Bayesian analysis, information on the choice of priors and Markov chain Monte Carlo settings

$\boldsymbol{x} \square$ For hierarchical and complex designs, identification of the appropriate level for tests and full reporting of outcomes

$\mathbf{x} \square$ Estimates of effect sizes (e.g. Cohen's $d$, Pearson's $r$ ), indicating how they were calculated

our web collection on statistics for biologists contains articles on many of the points above.

\section{Software and code}

Policy information about availability of computer code
Data collection
No commercial or open source databases used.

Data analysis

Short sequence read alignment using Burrows-Wheeler transform, Li \& Durbin. Bioinformatics 2009; 25: 1754; Genome consensus generated using Geneious ver 11.1.4; Percentages of nucleotides at each position of the genome using bam-readcount (https:// github.com/genome/bam-readcount)

For manuscripts utilizing custom algorithms or software that are central to the research but not yet described in published literature, software must be made available to editors/reviewers. We strongly encourage code deposition in a community repository (e.g. GitHub). See the Nature Research guidelines for submitting code \& software for further information.

\section{Data}

Policy information about availability of data

All manuscripts must include a data availability statement. This statement should provide the following information, where applicable:

- Accession codes, unique identifiers, or web links for publicly available datasets

- A list of figures that have associated raw data

- A description of any restrictions on data availability

GenBank accession numbers MT215193, MT215194, MT215195, MT270814, MT270815 and MT276600. Data will be released 1st May 2020

\section{Field-specific reporting}

Please select the one below that is the best fit for your research. If you are not sure, read the appropriate sections before making your selection. 


\section{Life sciences study design}

All studies must disclose on these points even when the disclosure is negative. Sample size $\quad \begin{aligned} & 15 \text { dogs, } 7 \text { cats and four human patients. All eligible subjects during study period } 10 \text { th Feb - 27th March } 2020 \text { were included. Sample size } \\ & \text { calculation is not relevant. }\end{aligned}$

Data exclusions No data exclusions

Replication $\quad$ RT-PCR assays have been done independently in two different laboratories with up to 6 different gene targets. All positive results were confirmed by re-extraction and repeat PCR on the original specimen.

Randomization Not relevant. An observational study. No intervention investigated

Blinding Not relevant. An observational study. No intervention investigated

\section{Reporting for specific materials, systems and methods}

We require information from authors about some types of materials, experimental systems and methods used in many studies. Here, indicate whether each material, system or method listed is relevant to your study. If you are not sure if a list item applies to your research, read the appropriate section before selecting a response.

\begin{tabular}{l|l|l}
\multicolumn{2}{l}{ Materials \& experimental sys } \\
\hline n/a & Involved in the study \\
\hline $\boldsymbol{x}$ & $\square$ Antibodies \\
\hline & $\mathbf{x}$ Eukaryotic cell lines \\
\hline $\boldsymbol{x}$ & $\square$ Palaeontology \\
\hline & $\boldsymbol{x}$ Animals and other organisms \\
\hline & $\boldsymbol{x}$ Human research participants \\
\hline & $\boldsymbol{x}$ Clinical data
\end{tabular}

\begin{tabular}{l|l}
\multicolumn{2}{l}{ Methods } \\
\hline n/a & Involved in the study \\
\hline $\boldsymbol{x}$ & $\square$ ChIP-seq \\
\hline $\boldsymbol{x}$ & $\square$ Flow cytometry \\
\hline $\boldsymbol{x}$ & $\square$ MRI-based neuroimaging
\end{tabular}

\section{Eukaryotic cell lines}

Policy information about cell lines

Cell line source(s)

Vero-E6 cells (ATCC CRL-1586)

Authentication

Cell lines obtained from ATCC. Original cell stocks maintained in liquid N2 storage and each thawed aliquot discarded after 20 cell passages.

Mycoplasma contamination

Confirmed to be free of mycoplasma using two methods. A cell culture based kit from Invivogen. PlasmoTest ${ }^{\text {TM }}$ - Mycoplasma Detection Kit and a PCR assay from ABM. https://www.abmgood.com/pcr-mycoplasma-detection-kit-g238.html

Commonly misidentified lines (See ICLAC register)

No commonly misidentified cell lines used.

\section{Animals and other organisms}

Policy information about studies involving animals; ARRIVE guidelines recommended for reporting animal research

Laboratory animals

Wild animals

Field-collected samples

Ethics oversight
None

None

Samples collected by veterinarians of the Department of Agriculture, Fisheries and Conservations as part of routine management of the animals

Agriculture, Fisheries and Conservation Department

Note that full information on the approval of the study protocol must also be provided in the manuscript. 


\section{Human research participants}

Policy information about studies involving human research participants

Population characteristics

Retrieving virus sequence data from four patients in contact with the dogs

\section{Recruitment}

Humans in two households diagnosed with COVID-19 in contact with the infected dogs.

Ethics oversight

Part of the routine public health epidemic response and also Institutional Review Board approval UW20-168, University of Hong Kong Hospital Authority of Hong Kong West Cluster.

Note that full information on the approval of the study protocol must also be provided in the manuscript.

\section{Clinical data}

Policy information about clinical studies

All manuscripts should comply with the ICMJEguidelines for publication of clinical research and a completedCONSORT checklist must be included with all submissions.

Clinical trial registration

Study protocol

Data collection

Outcomes
This is not a clinical trial

This is an emergency public health response during a pandemic.

Epidemiological data collected as part of routine outbreak investigation during the period 10th February - 27 th March 2020.

Relevant outcomes reported in manuscript. 